\title{
EFFECTS OF KINESIO TAPING IN A PHYSICAL THERAPIST WITH ACUTE LOW BACK PAIN DUE TO PATIENT HANDLING: A CASE REPORT
}

\author{
GAK HWANG-BO ${ }^{1}$ and JUNG-HOON LEE ${ }^{2}$ \\ ${ }^{1}$ Daegu University, Daegu, Republic of Korea \\ Department of Physical Therapy, College of Rehabilitation Science \\ ${ }^{2}$ Inje University, Pusan Paik Hospital, Busan, Republic of Korea \\ Department of Physical Therapy
}

\begin{abstract}
Objectives: The paper describes the case of a physical therapist with acute Low Back Pain (LBP) due to patient handling and the efficacy of Kinesio Taping (KT) around the trunk in the treatment of this occupational LBP. Materials and Methods: KT was applied around the trunk for 3 days, for an average of $10 \mathrm{~h} /$ day. Kinesio tape was applied with 130-140\% stretch to the rectus abdominis, internal oblique, erector spinae, and latissimus dorsi muscles, which are activated in the process of lifting. Results: Following the KT application, the 'Visual Analog Scale' and 'Oswestry Disability Questionnaire scores' gradually decreased and active trunk range of motion limited by the LBP increased progressively. The physical therapist no longer complained of LBP and was able to handle patients without any pain. Conclusions: Hence, continuous application of KT around the trunk may be a supplementary treatment method for acute LBP in physical therapists and enable continuous patient handling without any loss of work time due to occupational LBP. In addition, KT may also be applicable for the prevention and treatment of occupational LBP in other professions involving lifting heavy objects.
\end{abstract}

Key words:

Low back pain, Occupations, Patient lifting, Patient transfers

\section{INTRODUCTION}

Physical therapy (PT) practice involves continuous bending, lifting or transferring patients, and performing repetitive manual therapy techniques [1]. These elements increase the risk of work-related musculoskeletal disorders (WMSDs) in physical therapists [2]. However, the unique PT workplace culture to develop therapeutic goals may make it difficult for physical therapists to implement methods to minimize the risk of WMSDs [3]. Physical therapists have been shown to miss work time (32\%) [4] or leave the occupation (17-30\%) due to WMSDs [5]. Although many studies have assessed the prevalence of WMSDs among physical therapists, no study has examined appropriate measures of dealing with low back pain (LBP), which appears to be the most frequently reported WMSD in physical therapists.

Kinesio taping (KT) is a relatively new treatment technique, which is increasingly becoming an adjunctive method to treat musculoskeletal and neuromuscular problems [6]. However, no research has evaluated the

This work was supported by the Daegu University Research Grant, Grant No 20090239.

Received: Feb 17, 2011. Accepted: May 13, 2011.

Address reprint request to J.-H. Lee, Department of Physical Therapy, Inje University, Pusan Paik Hospital, 633-165, Gaegum-dong, Busanjin-gu, Busan 14-735, Republic of Korea (e-mail: dreampt@hanmail.net). 
effects of KT on occupational LBP. We describe the case of a physical therapist with acute LBP due to patient handling and the efficacy of KT around the trunk in the treatment of this occupational LBP.

\section{CASE REPORT}

A 36-year-old male physical therapist developed acute LBP while lifting a stroke patient in order to transfer him from his wheelchair to a mat. The physical therapist had treated patients with musculoskeletal and neuromuscular problems for 8 years. He did not have any history of LBP. In the initial assessment, a Visual Analog Scale (VAS) score (0 representing no pain, 10 representing the worst imaginable pain) of 8/10 was reported for the low back area. The Oswestry Disability Index (ODI) score (ranging from $0 \%$ [no disability] to 100\%), which is a validated instrument for measuring disability in spinal problems [7], was 72\%. The back range of motion (ROM) instrument, reliable for the measurement of lumbar mobility [8], showed trunk flexion of $8^{\circ}$ (normal range: $0-80^{\circ}$ ) [9], extension of $7^{\circ}$ (normal range: $0-30^{\circ}$ ) [9], right lateral flexion of $5^{\circ}$ (normal range: $0-35^{\circ}$ ) [9], left lateral flexion of $5^{\circ}$ (normal range: $0-35^{\circ}$ ) [9], right rotation of $5^{\circ}$ (normal range: $0-45^{\circ}$ ) [9], and left rotation of $5^{\circ}$ (normal range: $0-45^{\circ}$ ) (Table 1) [9].

\section{MATERIALS AND METHODS}

KT was applied around the trunk for 3 days, for an average of 10 h/day. Kinesio tape (Kinesio Tex; KT-X-050, Tokyo, Japan) was applied with 130-140\% stretch [10] to the rectus abdominis (RA), internal oblique (IO), erector spinae (ES), and latissimus dorsi (LD) muscles, which are activated in the process of lifting [11]. For the RA, KT was applied while the therapist was flexing both knees, from near the symphysis pubis to the xiphoid process and the 5th to 6th costal cartilages (Photo 1A). For the IO, KT was applied when the therapist was in the side-lying position,

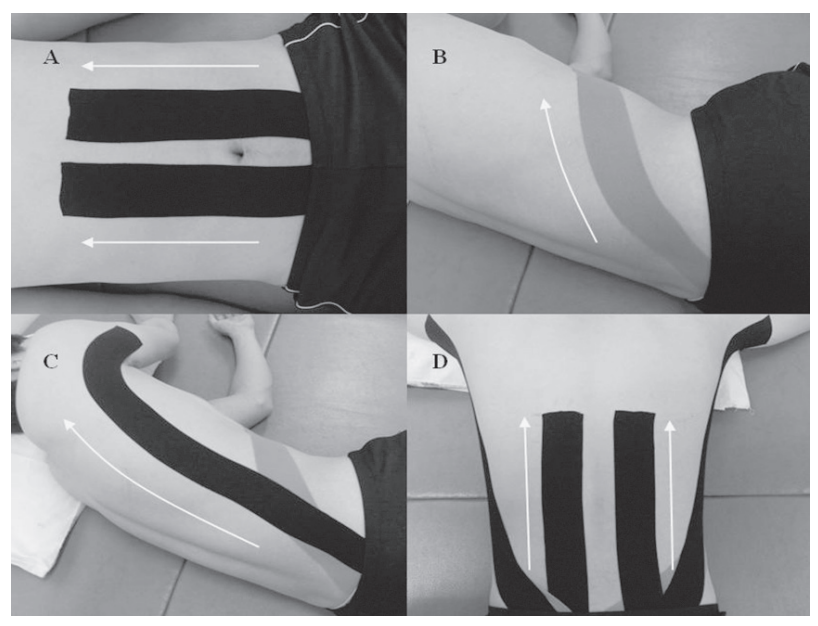

Photo 1. Kinesio taping (KT) application for the rectus abdominis (A), internal oblique (B), latissimus dorsi (C), erector spinae (D) muscles.

Table 1. The physical assessment results

\begin{tabular}{lccccc}
\hline \multicolumn{1}{c}{ Assessment } & 1st KT & After 1st KT & After 2nd KT & After 3rd KT & Final \\
\hline Visual Analogue Scale (VAS) & 8 & 5 & 3 & 2 & 0 \\
Oswestry Disability Index (ODI) score (\%) & 72 & 50 & 34 & 24 & 0 \\
Trunk flexion angle $\left({ }^{\circ}\right)$ & 8 & 30 & 55 & 75 & 80 \\
Trunk extension angle $\left({ }^{\circ}\right)$ & 7 & 15 & 21 & 27 & 28 \\
Trunk right lateral flexion angle $\left(^{\circ}\right)$ & 5 & 13 & 25 & 29 & 33 \\
Trunk left lateral flexion angle $\left({ }^{\circ}\right)$ & 5 & 12 & 18 & 25 & 35 \\
Trunk right rotation angle $\left(^{\circ}\right)$ & 5 & 27 & 33 & 40 & 50 \\
Trunk left rotation angle $\left(^{\circ}\right)$ & 5 & 25 & 36 & 40 & 45 \\
\hline
\end{tabular}

KT - Kinesio taping. 
from the anterior half of the crest to the linea alba by the aponeurosis (Photo 1B). For the LD and ES, KT was applied when the therapist was in the prone position, from the spinous process of the sacrum to the lesser tubercle of the humerus (Photo 1C) and from the medial crest of the sacrum to the inferior borders of the lower 6 or 7 ribs (Photo 1D), respectively. The therapist handled patients as before after KT was applied.

\section{RESULTS}

As shown in Table 1, during KT application for 3 days, the VAS and ODI scores gradually decreased and active trunk ROM increased progressively. In the final assessment, the VAS score had decreased to 0/10, the ODI score had decreased to $0 \%$, and trunk flexion had increased to $80^{\circ}$, extension to $30^{\circ}$, right lateral flexion to $33^{\circ}$, left lateral flexion to $35^{\circ}$, right rotation to $50^{\circ}$, and left rotation to $45^{\circ}$. The physical therapist no longer complained of LBP and was able to handle patients without any pain.

\section{DISCUSSION}

Most physical therapists continue working with WMSDs (95\%) [5], which most commonly affect the lower back [2]. The prevalence of LBP among physical therapists has been shown to range from $68 \%$ to $58 \%$ in the UK [4] and from $45 \%$ [12] to $62 \%$ [13] in the USA. Patient handling, including lifting and transferring, is associated with an increased risk of developing WMSDs of the lower back [2]. Lifting can generate large tension, compression, and shear forces at the base of the spine [11], and thus it is a risk factor for occupational LBP [2].

In this case study, KT application around the trunk for 3 days reduced acute LBP and increased trunk ROM. KT may improve joint instability, strengthen weakened muscles, reduce pain, and assist with postural alignment [6]. The elasticity of Kinesio Tape increases tension by stimulation during active trunk movement. Increased tension may facilitate pain inhibitory mechanisms (e.g., gate control theory) via afferent stimuli to large-diameter sensory nerve fibers, thereby alleviating nociception [14]. As the patient's pain level decreased, the fear of pain during trunk movement may also have decreased, thus improving trunk ROM [15]. In addition, the elasticity of Kinesio Tape improves muscle activity by strengthening weakened muscles [10]. During lifting, the increased intra-abdominal pressure obtained by KT during LA contraction provides stability to the lumbar spine and flexes the vertebral column [11]. Vigorous contraction of the IO results in a corset effect in the posterior lumbar region, thereby withstanding unwanted torsion caused by asymmetrical lifting [11]. Unilateral activity of the ES causes lateral flexion of the vertebral column [11]. The LD assists in the transfer of the external load from the arms to the trunk and plays a role in extension and rotation of the lower back [11].

Thus, KT application may improve the function of the RA, IO, ES, and LD muscles and gradually increase active trunk ROM. Hence, continuous application of KT around the trunk may be a supplementary treatment method for acute LBP in physical therapists and enable continuous patient handling without any loss of work time due to occupational LBP. In addition, KT may also be applicable for the prevention and treatment of occupational LBP in other professions involving lifting heavy objects. Future studies are necessary to generalize the clinical effects of KT around the trunk in occupational LBP.

\section{REFERENCES}

1. West DJ, Gardner D. Occupational injuries of physiotherapists in North and Central Queensland. Aust J Physiother 2001;47(3):179-83.

2. Campo M, Weiser S, Koenig KL, Nordin M. Work-related musculoskeletal disorders in physical therapists: a prospective cohort study with 1-year follow-up. Phys Ther 2008;88(7):608-19. 
3. Cromie JE, Robertson VJ, Best MO. Work-related musculoskeletal disorders and the culture of physical therapy. Phys Ther 2002;82(5):459-72.

4. Glover W, McGregor A, Sullivan C, Hague J. Work-related musculoskeletal disorders affecting members of the Chartered Society of Physiotherapy. Physiotherapy 2005;91(3):138-47.

5. Darragh AR, Huddleston W, King P. Work-related musculoskeletal injuries and disorders among occupational and physical therapists. Am J Occup Ther 2009;63(3):351-62.

6. Kaya E, Zinnuroglu M, Tugcu I. Kinesio taping compared to physical therapy modalities for the treatment of shoulder impingement syndrome. Clin Rheumatol 2011;30(2):201-7.

7. Kim DY, Lee SH, Lee HY, Lee HJ, Chang SB, Chung SK, et al. Validation of the Korean version of the oswestry disability index. Spine 2005;30(5):E123-7.

8. Kachingwe AF, Phillips BJ. Inter- and intrarater reliability of a back range of motion instrument. Arch Phys Med Rehabil 2005;86(12):2347-53.

9. Clarkson HM. Musculoskeletal assessment: joint range of motion and muscle strength. 2nd ed. Philadelphia: Lippincott Williams \& Wilkins; 2000.
10. Kase K, Wallis J, Kase T. Clinical therapeutic applications of

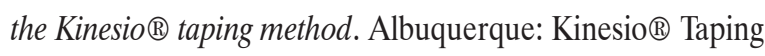
Association; 2003.

11. Neumann DA. Kinegiology of the musculoskeletal system: foundations for rehabilitation. 2nd ed. St Louis: Mosby; 2009.

12. Bork BE, Cook TM, Rosecrance JC, Engelhardt KA, Thomason ME, Wauford IJ, et al. Work-related musculoskeletal disorders among physical therapists. Phys Ther 1996;76(8): 827-35.

13. Holder N, Clark H, DiBlasio JM, Hughes CL, Scherpf JW, Harding L, et al. Cause, prevalence and response to occupational musculoskeletal injuries reported by physical therapists and physical therapy assistants. Phys Ther 1999;79(7):642-52.

14. Thelen MD, Dauber JA, Stoneman PD. The clinical efficacy of kinesio tape for shoulder pain: a randomized, double-blinded, clinical trial. J Orthop Sports Phys Ther 2008;38(7):389-95.

15. González-Iglesias J, Fernández-de-Las-Peñas C, Cleland JA, Huijbregts P, Del Rosario Gutiérrez-Vega M. Short-term effects of cervical kinesio taping on pain and cervical range of motion in patients with acute whiplash injury: a randomized clinical trial. J Orthop Sports Phys Ther 2009;39(7):515-21.

This work is available in Open Access model and licensed under a Creative Commons Attribution-NonCommercial 3.0 Poland License - http://creativecommons.org/ licenses/by-nc/3.0/pl/deed.en. 